\title{
Polymeric Photosensitizer Nano-drugs for Long Term Therapeutic Effect: in vitro and in vivo Phototoxicity against Human Colon Cancer (HCT-116)
}

\author{
Byoung-chan Bae and Kun $\mathrm{Na}^{*}$
}

\author{
Department of Biotechnology, The Catholic University of Korea, 43 Jibong-ro , Wonmi-gu, \\ Bucheon-si, Gyeonggi-do 420-743, Korea \\ kna6997@catholic.ac.kr
}

\begin{abstract}
Polymeric PS nano-drugs (chlorin e6 conjugate with acetylated hyaluronic acid and chlorin e6 conjugate with acetylated chondroitin sulfate; NPSs) were synthesized via carbodiimide reaction as nano-scale drugs for long term photodynamic therapy. The samples were self-assembled with average sizes below $200 \mathrm{~nm}$. Their self-photoquenching properties in the aqueous phase were observed, while fluorescence intensity was strongly correlated with the amount of Ce6 in the organic solvent dimethyl sulfoxide (DMSO). In vitro phototoxicities of the NPSs are similar with free Ce6 measured by 3-[4,5-dimethylthiazol-2- yl]-2,5-diphenyltetrazolium bromide (MTT) assay. The self-quenching effect and fluorescence recovery of NPSs were determined using KODAK imagestation. Compared with free Ce6, NPSs were retained at the tumor region due to their self-aggregate property. Also, in vivo tumor suppression effect was maintained until 10 days. These results suggest that the NPSs can give new idea for long term therapy in the clinical application of photodynamic therapy.
\end{abstract}

Keywords: polymeric photosensitizer, photodynamic therapy, long term therapy, biocompatible polymers

\section{Introduction}

Recently, photodynamic therapy (PDT) has been highlighted as a modality for the treatment of various diseases due to its unique advantages of noninvasive application and a rapid recovery. However, problems with low specificity to the target region, low solubility, high cost and shortterm period for re-treatment still remain [1]. To solve these problems, many researchers make increase the solubility of photosensitizer (PS) using various method. PS solubility was first increased via a conjugate with functional groups, such as carboxylic groups. However, these functionalized PSs were not still accepted for use by clinical application due to the low solubility and low retention time at the tumor region. Hence, various delivery systems for PSs have been investigated. The delivery systems have provided higher solubility of PSs in the aqueous phase, long term retention within the body, and even an increased specificity for tumors. So, we development polymeric PSs for soluble, injectable and long term therapeutic agent using soluble polymers such as hyaluronic acid (HyalA) and chondroitin sulfate (ChonS).

Hyaluronic acid (HyalA), the only nonsulfated glycosaminoglycan composed of repeating disaccharide units of N-acety- D glucosamine and D-glucuronic acid [2]. In addition, to control active targeting of tumor cells through the selective interaction between HyalA and its receptors. It is also known that HyalA specific receptors such as CD44 [35] overexpress on the surface of various tumor 
cells, enhancing the binding and internalization of HyalA. Furthermore, ChonS is composed of alternating units of (R-1,3)linked glucuronic acid and (R-1,4) Nacetyl galactosamine (GalNac), with sulfate at either the 4- or 6-position of GalNac [6]. Because it is found in cartilage, skin, corneas, extracellular matrix (ECM), ChonS possesses good biocompatibility $[7,8]$. In addition, ChonS also has the ability to bind to hyaluronic acid receptors overexpressed on cancer cells [3, 9]. which induces an effective tumor homing property [10]. The chlorin e6 (Ce6) used in this study emits a more stable fluorescence than pheophorbide a, which is affected considerably in aqueous solution [11].

In this paper, we report on the therapeutic effects the $\mathrm{Ce} 6$ and polymeric $\mathrm{Ce} 6$ against HCT116 cells, observed not only from in vitro experiments, but also from in vivo experiments.

\section{Experimental}

\subsection{Materials}

HyalA (MW 37,000 Da) was provided by the Bioland Company (Cheonan, Korea). ChonS (MW 50,000) was purchased from the Carl Roth Company (Germany). Ce6 was acquired from Frontier Scientific (Salt Lake City, UT). Acetic anhydride (AA), pyridine, 1,3-dicyclohexyl carbodiimide (DCC), 4-dimethylaminopyridine (DMAP), formamide and anhydrous dimethyl sulfoxide (DMSO) were obtained from Junsei Chemical. Dulbecco's modified Eagle's medium (DMEM), fetal bovine serum (FBS), antibiotics (penicillin/streptomycin), and Dulbecco's phosphate buffered saline (DPBS) were obtained from GIBCO BRL (Invitrogen, Carlsbad, CA). All other chemicals and solvents used were of analytical grade.

\subsection{Synthesis of Ac-HyalA/Ce6 and Ac- ChonS/Ce6}

Acetylation of HyalA (Ac-HyalA) and ChonS (Ac-ChonS) was performed in the same manner as that previously reported by our group [10]. In brief, to a stirred solution of $1 \mathrm{~g}$ of HyalA and ChonS in $10 \mathrm{ml}$ of formamide at room temperature, $557 \mu \mathrm{l}$ of pyridine and $500 \mu \mathrm{l}$ of AA were added. The reaction mixture was stirred for $12 \mathrm{~h}$ and was then transferred to a molecular porous dialysis membrane (Mw cutoff $3.5 \mathrm{kDa}$ ) for 3 days. The solution was lyophilized to obtain a yellowish powder. The structure of Ac-HyalA, Ac-ChonS and the degree of acetylation was analyzed by ${ }^{1} \mathrm{H}$ NMR spectroscopy. The solution of Ce6 in DMSO with the addition of predetermined amounts of DCC and DMAP was stirred for $1 \mathrm{~h}$ to activate the carboxylic groups, while Ac-HyalA and AcChonS was dissolved in DMSO via vigorous stirring, respectively. $10 \mathrm{mg}$ of Ce6 vs three 100 $\mathrm{mg}$ of HyalA and ChonS, mixed solutions were further stirred for $48 \mathrm{~h}$ at room temperature. The exhaustive dialysis (Mw cutoff $10 \mathrm{kDa}$ ) of the reactant mixture was conducted against distilled water (DW) for 3 days and was then repeated three times to remove unreacted free drug. The final solution was flash frozen and lyophilized. The amount of conjugated $\mathrm{Ce} 6$ was then analyzed using ${ }^{1} \mathrm{H}$ NMR spectroscopy.

\subsection{Physicochemical characterization of poly- meric PS nano-drugs}

The formation of polymeric PS nano-drugs (NPSs) were characterized by measuring the particle size and zeta potential using a dynamic light scattering (DLS) instrument (Zetasizer 3000, Malvern Instruments). For the measurement of hydrodynamic diameter and surface potential, NPSs in DW were prepared at a concentration of $1 \mathrm{mg} / \mathrm{ml}$ at room temperature. To monitor changes of photoactivity in different solvents, NPSs were dissolved in PBS and DMSO in EP-tubes, respectively. Fluorescence images were detected with KODAK Image Station after being dissolved in different solvents. Images were obtained using a 12-bit CCD camera (Image Station 4000 MM; Kodak, New Haven, CT, USA) equipped with a special $\mathrm{C}$-mount lens and a long wave emission filter (600-700 nm; Omega Optical, Brattleboro, VT, USA).

\subsection{In vitro photoactivity of Ce6 and NPSs}

HCT-116 cells $\left(1 \times 10^{4}\right.$ cells per well $)$ were seeded on 96-well plates and cultured in $200 \mu \mathrm{l}$ 
culture medium for 1 day. Two different samples of free Ce6 and NPSs were treated with various concentrations of Ce6 $(0 \mu \mathrm{g} / \mathrm{ml}$ to $2 \mu \mathrm{g} / \mathrm{ml})$. After $12 \mathrm{~h}$ of incubation, the medium was replaced with fresh RPMI 1640 just prior to irradiation at $2.0 \mathrm{~J} / \mathrm{cm} 2$ by a $670 \mathrm{~nm}$ laser source (Institute of Electronics). After $24 \mathrm{~h}$ of incubation, phototoxicity and dark toxicity were determined using 3-[4,5-dimethylthiazol-2-yl]3,5-diphenyltetrazolium bromide dye (MTT dye, $2 \mathrm{mg} / \mathrm{ml}$ ) uptake at $570 \mathrm{~nm}$ on an ELISA reader.

\section{5 in vivo time dependent fluorescence activity} of NPSs

All animal studies were approved by the Institutional Animal Care and Use Committee. Five-week-old male Balb/C-nu mice (Institute of Medical Science, Tokyo) were used for in vivo time dependent fluorescence activity of free Ce6 and NPSs. Free Ce6 and NPSs in $100 \mu 1$ of saline $(10 \mathrm{ug} / \mathrm{ml}$ of Ce6) were subcutaneously injected on the right back of the mice. The same mice were then scanned at a predetermined time. A $670 \mathrm{~nm}$ pulsed laser diode was used to excite Ce6 molecules. Long wave fluorescence emission $(600 \sim 700 \mathrm{~nm})$ was detected with a fast photomultiplier tube (Hamamatsu, Japan), and fluorescence images were obtained using a 12bit CCD camera (Image Station 4000 MM; Kodak, New Haven, CT) equipped with a special C-mount lens and a long wave emission filter (600 700 nm; Omega Optical, Brattleboro, VT, USA) [12]. Quantification of Ce6 intensity in images obtained at each time point was determined by comparison with the colored scale bar.

2.6 in vivo therapeutic effect of NPSs in tumorbearing mice

Subcutaneous tumors were established by xenograft HCT-116 cells $\left(\begin{array}{lll}1 & \mathrm{x} & 10^{6}\end{array}\right)$ into the backs of mice. When the tumor reached an average diameter of $\sim 6 \mathrm{~mm}$, free $\mathrm{Ce} 6$ and NP Ss $\left(10 \mu \mathrm{g} / \mathrm{cm}^{2}\right.$ of Ce6) were injected at the tumo $r$ region. Mice were divided into seven groups $t r$ eated with different injections, as follows: (1) sa line (the control group, $n=4),(2)$ free $\mathrm{Ce} 6$ witho ut irradiation $(n=4), \quad$ (3) Ac-
HyalA/Ce6 NPs without irradiation $(n=4),(4)$ Ac-ChonS/Ce6 NPs without irradiation $(n=4)$ (5) free Ce6 with irradiation $(n=4)$, (6) AcHyalA/Ce6 NPs with irradiation, (7) Ac-ChonSCe6 NPs with irradiation $(n=4)$. Irradiation was applied at $670 \mathrm{~nm}$ using a copper vapor laser (Institute of Electronics) for $100 \mathrm{~J} / \mathrm{cm}^{2}$. Two perpen dicular diameters of tumor were measured every two days using Vernier calipers (Mitutoyo Co., Japan), and the volume was calculated using the formula $\mathrm{V}=(\mathrm{a} \times(\mathrm{b} \times \mathrm{b})) / 2$, with $a$ being the largest and $b$ being the smallest diameter [13].

\section{Results and Discussion}

Our group has previously described the high AA modification for HyalA and ChonS to increase hydrophobicity, forming self-assembled Nano-drugs $[14,15]$. In this work, with a small feed of AA for the offer of hydrophobicity to provide only DMSO-soluble polymers, the low acetylation guaranteed that more functional groups on the HyalA and ChonS were available to the further conjugation according to the synthetic scheme illustrated in Scheme 1.
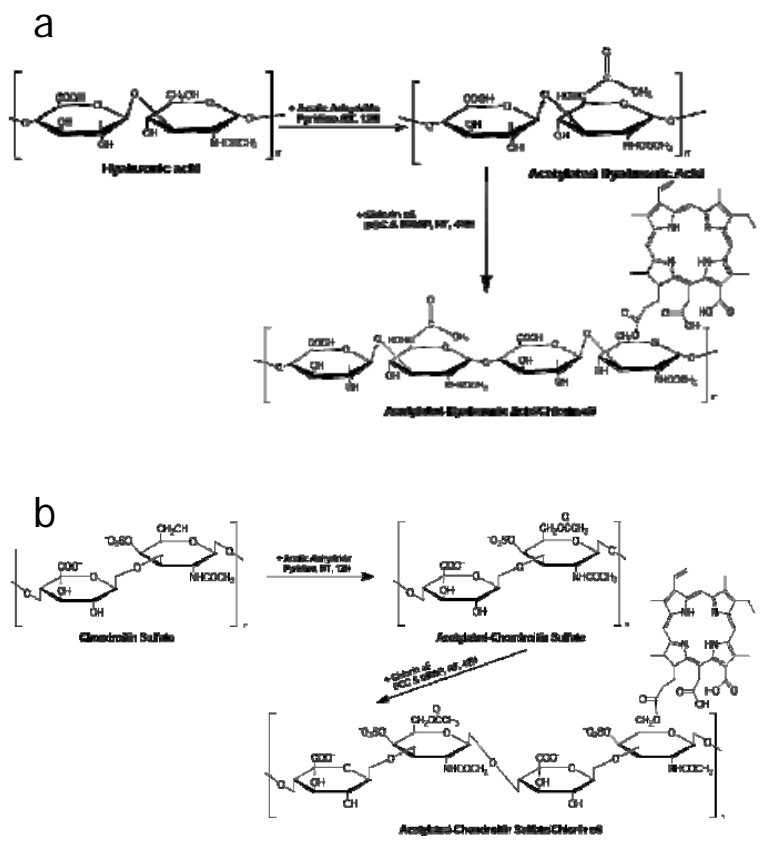

Scheme 1. Overall scheme of synthesis.

(a) Synthetic scheme of acetylated-Hyaluronic Acid/chlorin e6 (Ac-HyalA/Ce6) conjugates.

(b) Synthetic scheme of acetylatedchondroitin sulfate/chlorin e6 (AcChonS/Ce6) conjugates. 
The typical resonance of the acetyl moieties $(\delta \mathrm{H}$ 1.9) was identified to be superimposed on the signal from the acetamido methyl groups in disaccharide units of the HyalA and ChonS chain. Acetylated HyalA and ChonS (Ac-HyalA and Ac-ChonS) were synthesized with Ce6 through the conventional carbodiimide reaction [16] and the amounts of conjugated Ce6 were determined, as listed in Table 1, the structure of which were verified by ${ }^{1} \mathrm{HNMR}$. The characteristic peaks of $\mathrm{Ce} 6$ such as $10 \mathrm{H}, 5 \mathrm{H}$, and $20 \mathrm{H}$ were identified from $\delta 9.8$ to $\delta$ 8.7. Ce6 in Ac-HyalA/Ce6 and AcChonS/Ce6 were estimated by UV-Vis, which indicated that there were about 0.12 and 0.17 Ce6 moieties per polymer unit (two glucose units), respectively. Finally, when dispersed in an aqueous phase, NPSs self-assembled nanosized particles by anchoring the Ce 6 moieties to the inner core due to their strong hydrophobic interactions. The surrounding HyalA and ChonS chains play a critical role in providing high solubility and targeting abilities. To confirm the formation of NPSs, the mean diameters of each sample in PBS were measured by DLS as $185 \mathrm{~nm}, 165 \mathrm{~nm}$, respectively (Table 1). Negative potentials on the surface indicated that a HyalA and ChonS chains with a negative charge covered the self-assembled NPSs (Table $1)$.

Table 1. The Characteristics of NPSs in Aqueous Solution

\begin{tabular}{ccccc}
\hline & $\begin{array}{c}\text { Ce6 } \\
\text { conjugate } \\
\text { content }\end{array}$ & Size & $\begin{array}{c}\text { z- } \\
\text { potential } \\
(\mathbf{m V})\end{array}$ & PDI \\
\hline $\begin{array}{c}\text { Ac- } \\
\text { HyalA/Ce6 } \\
\text { Ac- }\end{array}$ & 0.12 & $185 \pm 15$ & -32 & 0.245 \\
ChonS/Ce6 & 0.17 & $162.5 \pm 10$ & -33 & 0.206 \\
\hline
\end{tabular}

${ }^{\mathrm{a}}$ Degree of substitution of $\mathrm{Ce} 6$ molecules per 2 glucose rings.

b Determined using a Zetasizer (Malvern Instruments) at a Nano-drug concentration of 1 $\mathrm{mg} / \mathrm{ml}$ in DW at $25^{\circ} \mathrm{C}$.

As can be seen from the data, the sizes of the three samples were similar, and all were below $200 \mathrm{~nm}$, which is known to lead to passive targeting and prolonged retention in tumors due to the enhanced permeation and retention (EPR) effect.

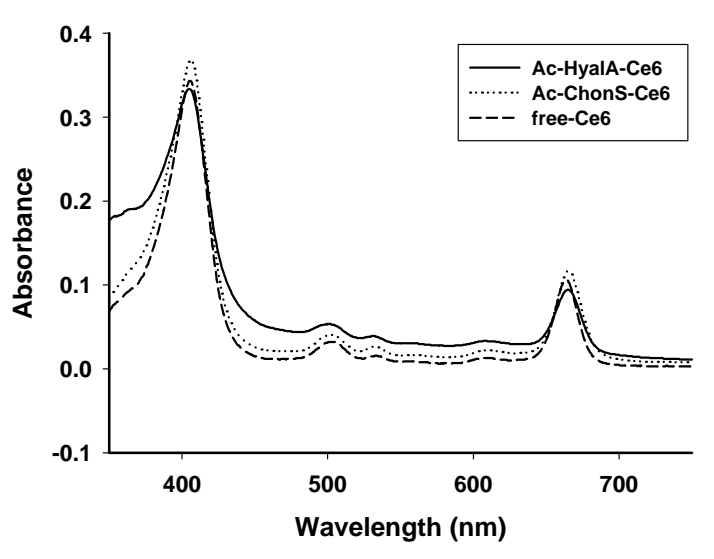

Figure 1. UV-Vis spectra of free Ce6 and NPSs in dimethyl sulfoxide (DMSO).

Also, the UV-Vis absorption spectrum of NPSs and free $\mathrm{Ce} 6$ showed similar results, despite chem ical modification of $\mathrm{Ce} 6$ molecules (Figure 1). To monitor photoactivity of NPSs and free Ce6 in different solvents were determined by KODAK Image Station.

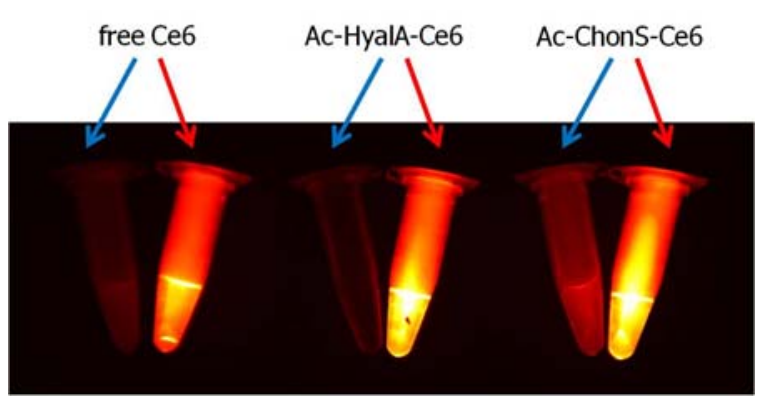

Figure 2. Bright and NIRF image of NPSs and free Ce6 in different solvent.(blue arrow: in PBS, red arrow: in DMSO)

Figure 2. shows the near-infra red fluorescence (NIRF) images Ac-HyalA/Ce6, Ac$\mathrm{ChonS} / \mathrm{Ce} 6$ and free $\mathrm{Ce} 6$ at a similar $\mathrm{Ce} 6$ concentration in DMSO or PBS. In PBS, due to the hydrophobic and $\pi-\pi$ stacking interactions of Ce6, Ac-HyalA/Ce6, Ac-ChonS/Ce6 and free Ce6 decreased photoactivity. Contrasting photoactivities were observed when AcHyalA/Ce6, Ac-ChonS/Ce6 and free $\mathrm{Ce} 6$ were dissolved in DMSO. The results implied the self-photoquenching potential of NPSs via selfassembly in the aqueous phase [17]. 


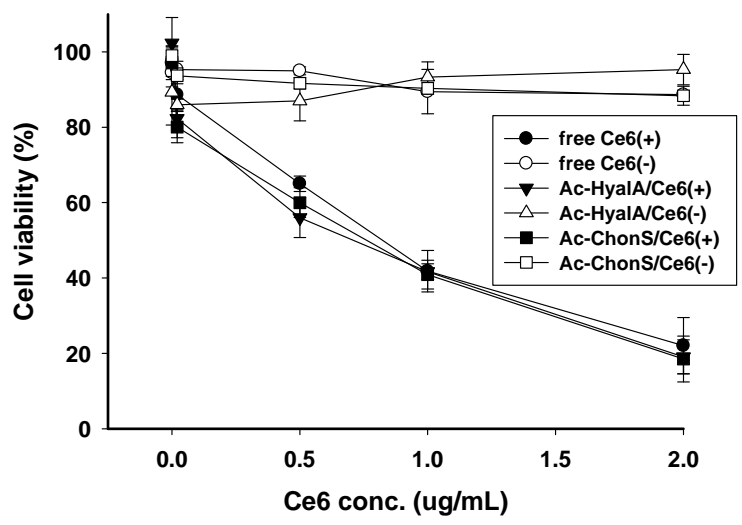

Figure 3. Cytotoxicity of different concentration of free $\mathrm{Ce} 6$ and NPSs.

In the experiment of in vitro phototoxicity determination against HCT-116 cells (Figure 3), little cytotoxicity was found in the NPSs and free Ce6 treated cells that were maintained in darkness. However, HCT-116 cells treated with light at an intensity of $2 \mathrm{~J} / \mathrm{cm}^{2}$ using different Ce6 concentrations $(0,0.5,1$, and $2 \mu \mathrm{g} / \mathrm{ml})$ showed enhanced cytotoxicity. Increase cytotoxicity according to $\mathrm{Ce} 6$ concentration were similar to those of Ac-HyalA/Ce6, AcChonS/Ce6 and free Ce6, resulting in an IC50 < 0.74 . Based on these results, in vivo experiments are required to further emphasize the advantage of NPSs. We suggest here the in vivo time dependent fluorescence activity of NPSs.

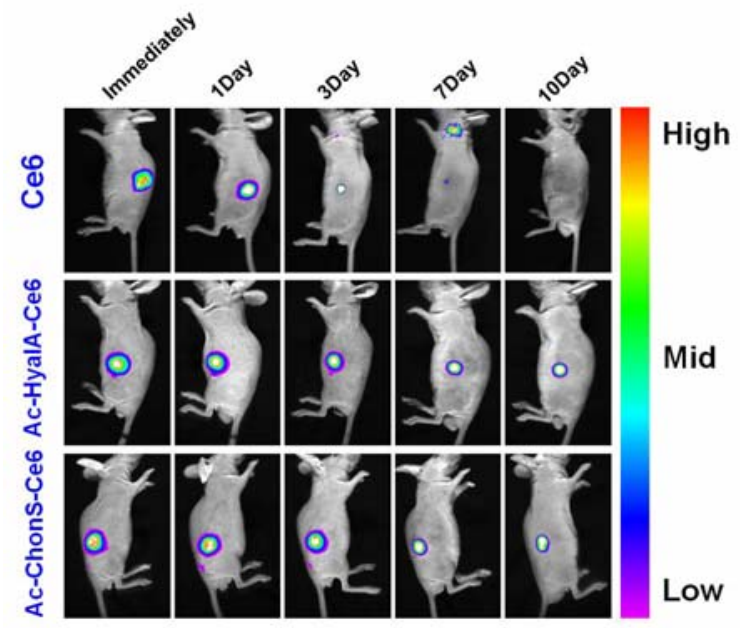

Figure 4. Time dependent in vivo fluorescence images of tumor xenografted balb/c-nude mice tumoral injected with free Ce6, Ac-HyalA/Ce6, and Ac-ChonS/Ce6 Nano-drug.
The time dependent photoactivities of AcHyalA/Ce6, Ac-ChonS/Ce6 and free Ce6 were monitored after being injected into the tumor region using tumor-bearing mice model. Immediately after injection, Ac-HyalA/Ce6, AcChonS/Ce6 and free Ce6 showed the strong florescent signal, which was maintained for 1 day. However, the fluorescent signal of free Ce6 injected mice gradually decreased after 3 days. Despite the presence of Ce6, NPSs are still maintained at tumor region for 10 days (Figure 4). The different retention time data from NPSs and free Ce6 treated mice showed substantial evidence of the long term therapeutic effects of

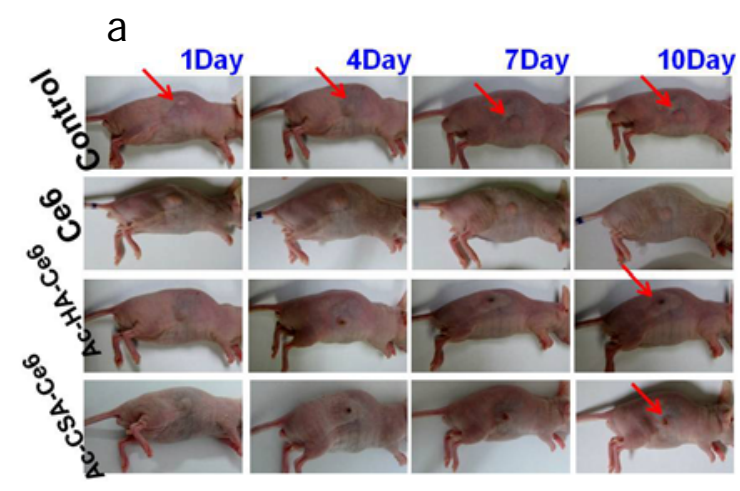

b

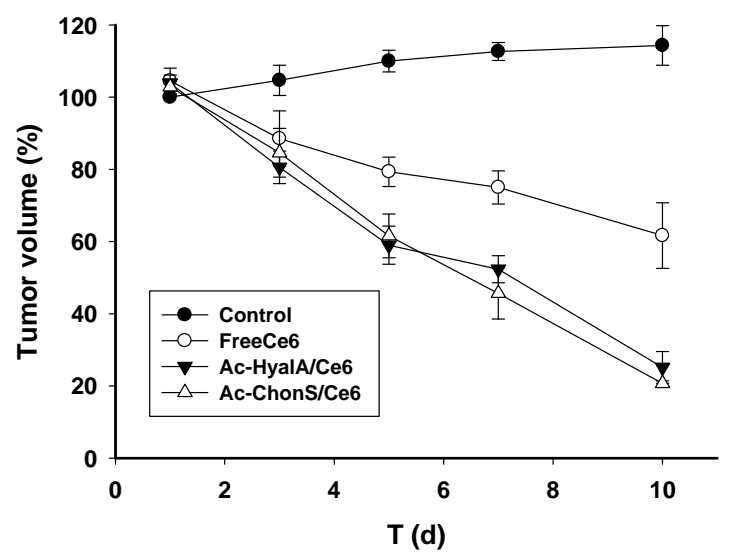

Figure 5. Time dependent in vivo fluorescence images of tumor xenografted balb/c-nude mice tumoral injected with free $\mathrm{Ce} 6, \mathrm{Ac}-\mathrm{HyalA} / \mathrm{Ce} 6$, and Ac-ChonS/Ce6 Nano-drug (a) Ac-HyalA/Ce6, AcChonS/Ce6 and free Ce6 (10 ug/ml of Ce6) in HCT116 tumor-bearing mice. Therapeutic efficacy was monitored for 10 days. (b) Tumor growth of HCT116 tumor-bearing mice with irradiation $(10 \mathrm{ug} / \mathrm{ml}$ of Ce6). Each tumor volume was measured for 10 days after post-treatment. All data represent the mean where $n=4$. 
the drug. On the basis of this result, we performed animal therapeutic experiments using tumor (HCT-116) xenografted mice. Balb/c nude tumor-bearing HCT-116 mice were subcutaneously injected with Ac-HyalA/Ce6, Ac-ChonS/Ce6 and free $\mathrm{Ce} 6$ and were then treated with $670 \mathrm{~nm}$ light for $100 \mathrm{~J} / \mathrm{cm}^{2}$.

Every 2 days for a period of 10 days the tumor sizes were measured using vernier calipers. Taken together, our results indicate that NPSs remained at the tumor region for 10days, unlike Ce6 and following irradiation, the NPSs produced cytotoxic singlet oxygen that induced a therapeutic effect on the tumors (figure $5 a, b$ ).

\section{Conclusion}

In this study, we developed a NPSs (AcHyalA/Ce6, Ac-ChonS/Ce6), which selfassembled when dispersed in aqueous solutions. The diameters of NPSs are $<200 \mathrm{~nm}$ with a mono-disperse size distribution. Photoactivity was disappeared in aqueous solution due to a self-quenching effect between PS molecules. In vitro cytotoxicity tests according to $\mathrm{Ce} 6$ concentration were similar to those of AcHyalA/Ce6, Ac-ChonS/Ce6 and free Ce6.

Unlike in vitro result, when the NPSs were subcutaneously tumoral injected into HCT-116 tumor-bearing mice, both the fluorescence signal and tumor suppression effect were maintained than free Ce6. The result is the evidence NPSs has advantages compared to free Ce6. Therefore, we conclude on the basis of these results, that the NPSs may be a potential new-generation photodynamic agent against small molecular PS.

\section{Ackowledgment}

This paper was financially supported by the Fundamental R\&D Program for Core Technology of Materials, Republic of Korea and the Korea Ministry of Education, Science and Technology through Strategic Research (2011-0028726).

\section{Reference}

1 R. Bonnett, Chemical Society Reviews, 24, (1995) 19-33.

2 J. Necas, L. Bartosikova, P. Brauner, J. Kolar, Veterinarni Medicina, 53, (2008) 397-411. 3 A. Aruffo, I. Stamenkovic, M. Melnick, C. B. Underhill, B. Seed, Cell, 61, (1990) 13031313.

4 M. Culty, H. A. Nguyen, C. B. Underhill, The Journal of cell biology, 116, (1992) 1055-1062.

5 C. UNDERHILL, Journal of cell science, 103, (1992) 293-298.

6 N. Volpi, Chondroitin sulfate: structure, role and pharmacological activity, Vol. 53, Academic Press, 2006.

$7 \quad$ D. A. Wang, S. Varghese, B. Sharma, I. Strehin, S. Fermanian, J. Gorham, D. H. Fairbrother, B. Cascio, J. H. Elisseeff, Nature materials, 6, (2007) 385-392.

8 N. Volpi, F. Maccari, Journal of Chromatography B, 834, (2006) 1-13.

9 T. Murai, N. Sougawa, H. Kawashima, K. Yamaguchi, M. Miyasaka, Immunology letters, 93, (2004) 163-170.

10 W. Park, S. Park, K. Na, Colloids and Surfaces B: Biointerfaces, 79, (501-508.

11 B. Roeder, H. Wabnitz, Journal of Photochemistry and Photobiology B: Biology, 1, (1987) 103-113.

12 S. J. Lee, K. Park, Y. K. Oh, S. H. Kwon, S. Her, I. S. Kim, K. Choi, H. Kim, S. G. Lee, Biomaterials, 30, (2009) 2929-2939.

13 M. M. Tomayko, C. P. Reynolds, Cancer chemotherapy and pharmacology, 24, (1989) 148-154.

14 F. Li, B. Bae, K. Na, Bioconjugate chemistry, 21, 1312-1320.

15 F. Li, K. Na, Biomacromolecules, 12, 1724-1730.

16 K. M. Park, H. C. Kang, J. K. Cho, I. J. Chung, S. H. Cho, Y. H. Bae, K. Na, Biomaterials, 30, (2009) 2642-2652.

17 B. Bae, K. Na, Biomaterials, 31, (6325-6335. 